\title{
Epidemiología, tratamiento y mortalidad en pacientes infectados por enterobacterias productoras de carbapenemasas: estudio retrospectivo
}

\author{
Epidemiology, treatment and mortality in infection by carbapenemase-producing \\ Enterobacteriaceae: retrospective study
}

\author{
Alba Antequera M. ${ }^{1}$, Carmen Sáez B. ${ }^{1}$, Marianela Ciudad S. ${ }^{1}$, María José García B. ${ }^{1}$, Berta Moyano V. ${ }^{1}$, Pablo Rodríguez C. ${ }^{1}$, Emilia Roy V. ${ }^{1}$ \\ María Aguilera G. ${ }^{1}$, Ester Alonso N. ${ }^{1}$, María José Cárdenas I. ${ }^{1}$, Sara Castro G. ${ }^{1}$, Diego Domingo G. ${ }^{2}$ y Ana Barrios B. ${ }^{1}$
}

\author{
${ }^{1}$ Servicio Medicina Interna-Infecciosas. Hospital Universitario La Princesa. Madrid, España. \\ bServicio de Microbiología. Hospital Universitario La Princesa. Madrid, España. \\ Financiación: ninguna \\ Conflictos de interés: ninguno \\ Recibido: (segunda versión) 3 de marzo de 2020 / Aceptado: 25 de mayo de 2020
}

\section{Resumen}

Introducción: Las enterobacterias productoras de carbapenemasas (EPC) suponen un reto para la salud pública y la práctica clínica. Objetivo: Analizar la epidemiología, el tratamiento y la mortalidad en pacientes infectados por EPC. Material y Métodos: Análisis retrospectivo de 163 pacientes infectados por EPC en un hospital universitario desde julio de 2013 a octubre de 2015. Resultados: Klebsiella pneumoniae fue aislada en $95,1 \%$ de los casos, y la mayoría de las carbapenemasas pertenecían al grupo OXA-48 (93\%). La adquisición fue nosocomial en 124 casos $(77 \%)$, asociada a cuidados sanitarios en $30(18,6 \%)$, y $7(4,3 \%)$ fueron de adquisición comunitaria. Las infecciones más frecuentes fueron las del tracto urinario $(48,4 \%)$ y las respiratorias (19,5\%). Aproximadamente, la mitad de los pacientes recibieron monoterapia antimicrobiana. La tasa de mortalidad a los 30 días fue de 23,3\%. El análisis multivariante identificó que la presencia de shock séptico al diagnóstico (OR 4,2; IC 95\% 1,5-11) estaba asociada de manera independiente con mayor mortalidad en el primer mes, sin lograr identificar asociación con el tratamiento antimicrobiano inapropiado. Discusión: Son necesarios más estudios para aclarar si el tratamiento antimicrobiano de las infecciones por EPC debe ser combinado o si podría ser suficiente la monoterapia en infecciones leves.

Palabras clave: enterobacterias; carbapenemasas; resistencia antimicrobiana; antibioterapia; mortalidad; nosocomial

\begin{abstract}
Background: Carbapenemase-producing Enterobacteriaceae (CPE) has become a significant problem in terms of public health and clinical outcome. Objective: To assess the epidemiology, treatment and mortality in patients with infection due to CPE. Material and Methods: A retrospective analysis of 163 patients with CPE infection was carried out in a university hospital from July 2013 to October 2015. Results: A total of 163 patients were included over the study period. Klebsiella pneumoniae was isolated in $95.1 \%$ of cases, and most of carbapenemases belonged to the OXA-48 group (93.0\%). Acquisition was nosocomial in 124 cases $(77.0 \%)$, healthcare-associated in $30(18.6 \%)$, and 7 cases $(4.3 \%)$ were community-acquired. The most frequent infections identified in this study were urinary tract (48.4\%) and respiratory (19.5\%) infections. Approximately half of the patients received antibiotic monotherapy. The 30-day mortality rate was $23.3 \%$. Multivariate analysis revealed that the presence of septic shock at diagnosis (OR 4.2; IC 95\% 1.5-11) was independently associated with an increase in death during the first month, unable to identify association with inappropriate antibiotic treatment. Discussion: Further studies are needed to clarify whether antibiotic treatment of EPC infections should be combined or if monotherapy might be sufficient in mild infections.

Keywords: Enterobacteriaceae; carbapenemase; antibiotic resistance; antibiotic therapy; mortality; nosocomial.
\end{abstract}




\section{Introducción}

L as enterobacterias representan uno de los principales grupos de patógenos involucrados, tanto en las infecciones adquiridas en la comunidad, como en las relacionadas con los cuidados sanitarios ${ }^{1}$. El desarrollo de resistencias a carbapenémicos supone un desafío clínico y de salud pública de dimensiones mundiales. Grecia y E.U.A. realizaron las primeras notificaciones de aislamiento de cepas de enterobacterias productoras de carbapenemasas (EPC) al inicio de la década de los $2000^{2}$. Posteriormente, se produjo una rápida expansión por el resto de países. De acuerdo con las estimaciones del Centro Europeo para la Prevención y el Control de las Enfermedades en el año 2018, la resistencia a carbapenémicos de Klebsiella pneumoniae entre los países de la Unión Europea, se situaba en torno a 7,5\%, con cifras entre 0 y $63,9 \%$ según los países, situándose la mayoría de ellos en niveles inferiores a 1\% ${ }^{3}$. En España, los primeros aislamientos de EPC se remontan al $2005^{4}$. Desde entonces, se han identificado casos aislados y brotes $^{5-8}$, siendo $K$. pneumoniae la especie más frecuente $(74,4 \%)^{7,9}$.

Las enterobacterias presentan mecanismos heterogéneos de adquisición de resistencia a carbapenémicos. Resumidamente, pueden estar mediados a través de la producción de carbapenemasas (EPC), mediante la producción de $\beta$-lactamasas de espectro extendido (BLEE) y/o cefalosporinasa $\mathrm{AmpC}$, combinadas con alteraciones en la permeabilidad de la membrana celular ${ }^{10}$. Se han descrito como factores de riesgo asociados a EPC: la edad, la gravedad de la enfermedad, el ingreso en Unidad de Cuidados Intensivos (UCI), el empleo previo de antimicrobianos (principalmente carbapenémicos, quinolonas y cefalosporinas) y los procedimientos invasivos (endoscopia, duración del catéter venoso) ${ }^{11}$.

Las EPC suponen un reto clínico. En primer lugar, las infecciones graves por estas bacterias se han asociado a altas tasas de mortalidad, con cifras que oscilan entre 40 y $80 \%{ }^{12,13}$. La mortalidad se ha asociado con las comorbilidades del paciente, la presencia de sepsis grave o shock séptico en la presentación, la gravedad del episodio, el origen diferente del urinario o biliar, y el tratamiento antimicrobiano, empírico y dirigido, inapropiados. En segundo lugar, suponen una dificultad terapéutica debido a las opciones limitadas para configurar un régimen antimicrobiano activo, puesto que habitualmente su sensibilidad se reduce a colistina, tigeciclina, ciertos aminoglucósidos y meropenem cuando la CIM es $\leq 8 \mu \mathrm{g} / \mathrm{mL}$. A ello se añade la escasez de nuevos antimicrobianos en desarro$1 \mathrm{l}^{14}$. Así mismo, aún se debate si el tratamiento debe ser siempre combinado con dos antimicrobianos o en algunos tipos de infección; por ejemplo, si en la infección urinaria podría ser suficiente un solo antimicrobiano.
En este trabajo analizamos la epidemiología de los aislamientos de EPC, así como los aspectos asociados a infecciones producidas por EPC, y sus resultados sobre la mortalidad en una cohorte de pacientes adultos de un hospital universitario de tercer nivel en España.

\section{Materiales y Métodos}

\section{Diseño del estudio y escenario}

Realizamos un estudio de cohortes, retrospectivo, en el Hospital Universitario de La Princesa, en la Comunidad de Madrid, con un área sanitaria de 310.000 personas, entre julio de 2013 y octubre de 2015. Analizamos las características demográficas, microbiológicas y clínicas de todos los aislamientos consecutivos de EPC durante dicho período.

\section{Variables y definiciones}

Los datos epidemiológicos y clínicos fueron recogidos en una base de datos informatizada específica. El criterio de inclusión fue todo paciente con al menos un cultivo positivo a EPC durante el período de reclutamiento. Sólo se incluyó el primer aislado de EPC en cada paciente, en una muestra clínica tomada por sospecha de infección. No se incluyeron las muestras epidemiológicas para descartar colonización.

Definición de infección o de colonización: Se estableció de acuerdo con la actitud del equipo médico a cargo del paciente en la historia clínica. Se consideró colonización/ contaminación si no se pautó tratamiento antimicrobiano activo frente al microorganismo.

Las variables analizadas incluyeron: características demográficas, co-morbilidades, lugar de adquisición (nosocomial, asociada a cuidados sanitarios o comunitaria), procedimientos invasivos en el año previo (colocación de catéter vesical u otro catéter urológico, catéter venoso central o dispositivos cardiacos, necesidad de ventilación mecánica, realización de endoscopias, hemodiálisis, prótesis valvular, colocación de prótesis articular o material de osteosíntesis, prótesis biliares o derivaciones ventriculares), y antimicrobiano durante al menos dos días en el año previo.

En el episodio de infección, se consideró el tipo de infección de acuerdo con las definiciones del $\mathrm{CDC}^{15}$, la presencia o no de bacteriemia, el servicio de origen de la muestra (críticos, especialidades médicas y quirúrgicas), la presencia o no de shock séptico asociado, el ingreso en UCI, la sensibilidad a antimicrobianos de la EPC, la terapia antimicrobiana empírica y dirigida (apropiada o no), la mortalidad global por cualquier causa durante el seguimiento y durante el episodio (a los 30 días), y la 


\section{Análisis estadístico}

El análisis estadístico se realizó con el programa esdel investigador como el fallecimiento a consecuencia de la infección por EPC, excluidas otras opciones menos probables).

La infección se clasificó según el lugar de adquisición, de acuerdo con los criterios de Friedman ${ }^{16}$. Tipo de infección: Se estableció de acuerdo con la impresión del equipo médico a cargo del paciente en la historia clínica. Antibioterapia apropiada: Se consideró si inclúa al menos un fármaco con actividad in vitro de acuerdo con los puntos de corte recomendados por EUCAST (European Committee on Antimicrobial Susceptibility Testing) ${ }^{17}$. Se consideró antimicrobiano plenamente activo a aquel para el que el microorganismo resultó sensible según la CIM en el antibiograma. Si la sensibilidad era intermedia se consideró antimicrobiano menos activo. En el caso de meropenem, a pesar de los datos de sensibilidad in vitro (sensible si CIM $\leq 2 \mu \mathrm{g} / \mathrm{mL}$, sensibilidad intermedia si CIM de $3-6 \mu \mathrm{g} / \mathrm{mL}$ y resistente si CIM $\geq 8 \mu \mathrm{g} / \mathrm{mL}$ ), se consideró plenamente activo si la CIM era $\leq 8 \mu \mathrm{g} / \mathrm{mL}$, por la posibilidad de tratamiento con altas dosis en estos casos. La antibioterapia recibida antes de disponer de los datos sobre sensibilidad se consideró empirica, y la posterior como definitiva. Se valoró la mortalidad como el fallecimiento por cualquier causa en los 30 días siguientes al día de la toma de la muestra diagnóstica, según constaba en los sistemas de información hospitalarios y de Atención Primaria.

\section{Estudios microbiológicos}

Las bacterias se identificaron mediante Microscan Walkaway ${ }^{\circledR}$ (Beckman Coulter, España) y se confirmaron mediante MALDI-TOF ${ }^{\circledR}$ (Bruker Daltonics). La sensibilidad antimicrobiana se determinó mediante el método de microdilución en caldo utilizando el sistema automatizado Microscan Walkaway ${ }^{\circledR}$ (Beckman Coulter, España) de acuerdo con las recomendaciones del fabricante. Todas las cepas con CIM alta o ligeramente alta a alguno de los tres carbapenémicos probados se incluyeron en el estudio, siguiendo las recomendaciones del EUCAST: $>0,125 \mu \mathrm{g} / \mathrm{mL}$ a meropenem y ertapenem $\mathrm{y}>1 \mu \mathrm{g} / \mathrm{mL}$ a imipenem ${ }^{18}$. La detección fenotípica de carbapenemasas se realizó en las cepas aisladas en 2013 y 2014 mediante el método colorimétrico Carba $\mathrm{NP}^{19}$ y su identificación genotípica mediante reacción de polimerasa en cadena (RPC), realizada en el Centro Nacional de Microbiología, Instituto de Salud Carlos III, Majadahonda. En 2015, las cepas fueron estudiadas fenotípicamente mediante el test de inmunocromatografía OXA-48 Card letitest ${ }^{\circledR}$ (Coris BioConcept, Bélgica) ${ }^{20}$, realizándose en las que se obtuvo un resultado negativo, la identificación genotípica mediante RPC en tiempo real con la técnica Xpert Carba- $\mathrm{R}^{\circledR}$ (Cepheid Sunnyvale, CA, E.U.A. $)^{21}$. tadístico SPSS (versión 23.0, Chicago, IL, E.U.A.). Para describir las características demográficas y clínicas de la muestra se presentan frecuencias absolutas y relativas para variables cualitativas y mediana y rango intercuartil (RIC) para variables cuantitativas. Se estudiaron los factores de riesgo para mortalidad (a 30 días), mediante un análisis univariante. Las variables estadísticamente significativas $(p<0,1) y / o$ con especial interés clínico se incluyeron en un análisis de regresión logística multivariante, con el objetivo de encontrar un modelo predictor de mortalidad. Se buscó ajustar un modelo parsimonioso, con el menor número de variables necesario, teniendo en cuenta el número de eventos para evitar la sobresaturación del modelo. Como medida de la capacidad predictora del modelo se calculó el área bajo la curva ROC. Todos los test se consideraron bilaterales y se consideró significación estadística un nivel de $\mathrm{p}<0,05$.

\section{Ética}

Este estudio se realizó respetando los acuerdos de la Declaración de Helsinki ${ }^{18}$. En cuanto a la revisión de las historias clínicas, se respetó la confidencialidad de los pacientes y se veló por el cumplimiento de la Ley Orgánica 15/1999 de Protección de Datos de Carácter Personal, así como el reglamento 1720-2007 que la desarrolla ${ }^{19}$.

\section{Resultados}

Identificamos 220 pacientes con muestras de aislamiento de EPC, considerándose como casos de infección 163 pacientes. Las características epidemiológicas y clínicas más relevantes de los casos de infección son presentados en las Tablas 1 y 2 .

La mediana (RIC) de tiempo de seguimiento fue de 4,5 (1-14) meses.

El servicio con mayor número de casos de aislamiento de EPC fue Medicina Interna-Infecciosas (24,4\%, n: 39), destacando que $7,4 \%$ (n: 12 ) de las muestras de pacientes con infección activa procedían del ámbito ambulatorio. Más de la mitad de los pacientes (58,2\%, n: 92$)$ habían sido hospitalizados en el año previo, más frecuentemente en los tres meses previos al aislamiento actual $(62,0 \%$, n: 57). y el dispositivo invasivo predominante fue el catéter urinario. Respecto al tipo de infección, los cuadros de infección urinaria supusieron casi la mitad de los casos $(48,4 \%, n: 77)$, y una cuarta parte de los pacientes presentaron bacteriemia $(25,5 \%, \mathrm{n}: 41)$.

Klebsiella pneumoniae fue la EPC aislada con mayor frecuencia $(95,1 \%$, n: 155$)$, seguida por Enterobacter
La co-morbilidad más frecuente fue diabetes mellitus 
Tabla 1. Características basales de los pacientes con infección por EPC (n: 163)

Sexo: Varones

$62,6 \%(102 / 163)$

Edad (años), mediana (RIC):

$77(64-85)$

$>80$ años

$38,7 \%(63)$

$>90$ años

$8,0 \%(13)$

Ingresos en el último año ( $\mathrm{n}$ : 158; desconocido n: 5)

$58,0 \%(92 / 158)$

Ingresos en los últimos 3 meses

$62,0 \%(57 / 92)$

Ingreso previo en Unidad de Pacientes Críticos en el último año (n: 154; desconocido n: 9)

$15,6 \%(24 / 154)$ Ingreso en los últimos 3 meses

$79,2 \%(19 / 24)$

Aislamiento previo de carbapenemasa en el último año

$14,1 \%(23 / 163)$

Aislamiento en últimos 3 meses

$91,3 \%(21 / 23)$

Tratamiento antimicrobiano previo en el último año ( $\mathrm{n}$ : 152; desconocido n: 11)

$84,9 \%(129 / 152)$

Tratamiento antimicrobiano en los últimos 3 meses ( $n$ : 116; desconocido n:13)

$91,4 \%(106 / 116)$

Presencia de co-morbilidades (n: 160; desconocido n: 3)

$96,3 \%(154 / 160)$

Diabetes mellitus

$31,3 \%(50)$

$27,5 \%(44)$

Cirugía previa

$19,4 \%(31)$

Uropatía/infección de tracto urinario

$15,0 \%(24)$

Insuficiencia cardiaca

$17,5 \%(28)$

Enfermedad pulmonar obstructiva crónica

$10,6 \%(17)$

$23,8 \%(38)$

Enfermedad renal crónica/hemodiálisis

$23,8 \%(38)$

Inmunosupresión

$27,5 \%(44)$

Enfermedad hematológica/oncológica

$3(2-4)$

$\mathrm{N}^{\circ}$ co-morbilidades, mediana (RIC)

$85,6 \%(125 / 146)$

Procedimiento invasivo (n: 146; desconocido n: 17)

$67,1 \%(98 / 146)$

EPC: enterobacterias productoras de carbapenemasas. RIC: rango intercuartil. SV: sondaje vesical

\section{Tabla 2. Análisis descriptivo de los episodios de infección por EPC (n: 163)}

Lugar de adquisición: (n: 161; desconocido n: 2)

Nosocomial

$77,0 \%(124 / 161)$

Asociada a cuidados sanitarios

$18,6 \%(30 / 161)$

Comunitaria

$4,3 \%(7 / 161)$

Origen de la infección: (n: 159; desconocido n: 4)

Urinario

$48,4 \%(77 / 159)$

Respiratorio

$19,5 \%(31 / 159)$

Abdominal

$12,6 \%(20 / 159)$

Piel y tejidos blandos

$11,9 \%(19 / 159)$

Endovascular (catéter)

$3,8 \%(6 / 159)$

Otros

$3,8 \%(6 / 159)$

Bacteriemia asociada (n: 161; desconocido n: 2)

$25,5 \%(41 / 161)$ 
Servicio de origen de la muestra: (n: 160; desconocido n: 3)

Críticos

$21,3 \%(34 / 160)$

Especialidades médicas

$53,1 \%(85 / 160)$

Medicina Interna/Infecciosas

$24,4 \%(39 / 160)$

Especialidades quirúrgicas

$25,6 \%(41 / 160)$

Sensibilidad a antimicrobianos (CIM $\mu \mathrm{g} / \mathrm{mL}$ ): (no disponible la CIM para todos los antimicrobianos en todas las muestras)

Ceftazidima ( $\leq 4)$

$12,3 \%(20 / 162)$

$5,0 \%(8 / 161)$

Piperazilina/tazobactam $(\leq 16)$

$6,7 \%(11 / 155)$

Ertapenem $(\leq 1)$

$63,8 \%(104 / 162)$

Imipenem $(\leq 8)$

$63,3 \%(69 / 109)$

Meropenem $(\leq 8)$

$29,4 \%(48 / 163)$

Gentamicina $(\leq 4)$

$96,6 \%(141 / 146)$

Amikacina $(\leq 16)$

$5,6 \%(9 / 162)$

Ciprofloxacina $(\leq 1)$

$58,6 \%(95 / 162)$

Cotrimoxazol $(\leq 4)$

$34,6 \%(36 / 104)$

Fosfomicina $(\leq 32)$

$87,2 \%(95 / 109)$

Tigeciclina $(\leq 2)$

$88,9 \%(8 / 9)$

Colistina $(\leq 2)$

Tratamiento antimicrobiano empírico apropiado o no: (n: 128; desconocido n: 35)

Incluye 2 (o más) ATM plenamente activos

$3,1 \%(4 / 128)$

Incluye 1 ATM plenamente activo y otro menos activo

$0,8 \%(1 / 128)$

Incluye sólo 1 ATM plenamente activo

$27,3 \%(35 / 128)$

Ningún ATM plenamente activo

$68,8 \%(88 / 128)$

Tratamiento antimicrobiano definitivo apropiado o no: (n: 144, desconocido/sin tto n: 19)

Incluye 2 (o más) ATM plenamente activos

$20,8 \%(30 / 144)$

Incluye 1 ATM plenamente activo y otro menos activo

$8,3 \%(12 / 144)$

Incluye sólo 1 ATM plenamente activo

$56,3 \%(81 / 144)$

Ningún ATM plenamente activo

$14,6 \%(21 / 144)$

Tipo de tratamiento antimicrobiano definitivo: ( $\mathrm{n}$ : 151; desconocido/sin tto n: 12)

Monoterapia

$52,9 \%(80 / 151)$

Carbapenem

$8,1 \%(12)$

Aminoglucósido

$18,8 \%(28)$

Tigeciclina

$6,7 \%(10)$

Cotrimoxazol

$6,0 \%(9)$

Otros

$26,2(21)$

Tratamiento combinado*

$47,0 \%(71 / 151)$

Carbapenem + aminoglucósido

$16,1 \%(24)$

Carbapenem + tigeciclina

$4,7 \%(7)$

Carbapenem + colistina

$2,0 \%(3)$

Tigeciclina + aminoglucósido

$8,1 \%(12)$

Colistina + aminoglucósido

$2,7 \%(4)$

$6,0 \%(9)$

Utras combinaciones con carbapenem

$8, \%(12)$

Shock séptico

$22,1 \%(36 / 163)$

Ingreso en Unidad de Pacientes Críticos

$33,1 \%(54 / 163)$

Exitus letalis

$50,3 \%(82 / 163)$

Exitus letalis relacionado con la infección (n: 61; no evaluables n: 21)

$27,9 \%(17 / 61)$

Mortalidad a 30 días

$23,3 \%(38 / 163)$

Episodio posterior de infección (en los siguientes 6 meses)

$14,7 \%(24 / 163)$

EPC: enterobacterias productoras de carbapenemasas. CIM: concentración inhibitoria mínima. ATM: antimicrobiano. Tto: tratamiento

* Tratamiento combinado incluye 3 combinaciones triples: piperazilina/tazobactam + amikacina + imipenem, meropenem + amikacina

+ colistina, o meropenem + tigeciclina + fosfomicina. 
cloacae (4,9\%, n: 8). El tipo de carbapenemasa (no disponible en 20 pacientes) predominante fue OXA-48 $(93,0 \%$, n: $133 / 143)$, seguido por VIM $(3,5 \%, n$ : 5$)$. En $36,7 \%$ de los pacientes, la CIM para meropenem de la cepa de EPC fue mayor de $8 \mu \mathrm{g} / \mathrm{mL}$.

La antibioterapia empírica fue considerada apropiada en $31,3 \%$ de los pacientes (n: 40/128) y los esquemas definitivos en $85,4 \%$ (n: 123/144). Según la adquisición de la infección fuera comunitaria o no (nosocomial o asociada a cuidados sanitarios), el tratamiento empírico fue apropiado en $16,7 \%(1 / 6) v s 32,2 \%(39 / 121)(\mathrm{p} 0,4)$ y el definitivo en 50\% (3/6) vs 87,6\% (120/137), respectivamente (p 0,03). Según la presentación con shock séptico o no, el tratamiento empírico fue apropiado en 44,0\% $(11 / 25)$ vs $28,1 \%(29 / 103)$ de los pacientes $(\mathrm{p} 0,13)$ y el definitivo en $89,3 \%(25 / 28)$ y $84,4 \%(98 / 116)$ (p 0,5$)$, respectivamente. Un $52,9 \%$ (n: 80/151) de los pacientes recibieron monoterapia en el esquema definitivo, mientras que 47,0\% (n: 71/151) recibieron un régimen combinado, cuyos fármacos más frecuentes fueron carbapenémico más aminoglucósido $(15,9 \%, \mathrm{n}: 24 / 151)$, seguido por tigeciclina más aminoglucósido $(7,9 \%, \mathrm{n}: 12 / 151)$. De los pacientes con infección urinaria (18 con bacteriemia) con tratamiento definitivo evaluable (73/77), 65,8\% (48/73) recibió monoterapia (aminoglucósidos los antimicrobianos más frecuentes), comparado con 42,1\% (32/76) en otros orígenes de la infección (p 0,005). De los pacientes con bacteriemia con tratamiento definitivo evaluable (39/41), 69,2\% ${ }^{27}$ recibió tratamiento antimicrobiano combinado frente a $39,3 \%$ de aquellos sin bacteriemia (p 0,002). En los pacientes con shock séptico (31/151 pacientes evaluables), el tratamiento fue combinado en $77,4 \%(24 / 31)$ frente a $39,2 \%(47 / 120)$ en los pacientes menos graves $(\mathrm{p}<0,001)$. Entre los ingresados en UCI, $62,7 \%$ recibió tratamiento combinado $(32 / 51)$ frente a $39 \%(39 / 100)$ de los no ingresados en UCI ( p 0,006).

Respecto a la mortalidad por cualquier causa, en nuestra cohorte a lo largo del seguimiento (mediana de 4,5 meses), encontramos una tasa de 50,3\% (n: 82). La mortalidad a los 30 días del aislamiento fue de $23,3 \%$ (n: 38). Se realizó un análisis univariante y multivariante para identificar posibles variables relacionadas con la mortalidad en los primeros treinta días entre los pacientes con infección (Tabla 3). Debido a la heterogeneidad de los regímenes antimicrobianos, no fue posible compararlos. El modelo final multivariante para la mortalidad en el

Tabla 3. Factores asociados con mortalidad a los 30 días entre los pacientes con infección por EPC

\begin{tabular}{|c|c|c|c|c|c|}
\hline & $\begin{array}{c}\text { Muerte a } 30 \text { días } \\
(\mathrm{n}=38 / 163 ; 23 \%) \mathrm{n}(\%)\end{array}$ & $\begin{array}{l}\text { Univariante } \\
\text { OR (IC 95\%) }\end{array}$ & p & $\begin{array}{l}\text { Multivariante } \\
\text { OR (IC 95\%) }\end{array}$ & $p$ \\
\hline Edad (años), mediana (RIC) & $\begin{array}{c}81 \text { (71-87) } \\
\text { Vivos: } 76(62-84)\end{array}$ & $1,03(1-1,1)$ & 0,06 & $1,02(0,99-1,1)$ & 0,2 \\
\hline $\begin{array}{l}\text { Co-morbilidad } \\
\text { Sí } \\
\text { No }\end{array}$ & $\begin{array}{c}36 / 154(23,4 \%) \\
1 / 6(16,7 \%)\end{array}$ & $\begin{array}{l}1,5(0,2-13) \\
\text { Referencia }\end{array}$ & 0,7 & & \\
\hline $\begin{array}{l}\text { Origen de la infección } \\
\text { Urinario } \\
\text { Otros }\end{array}$ & $\begin{array}{l}20 / 77(26 \%) \\
18 / 82(22 \%)\end{array}$ & $\begin{array}{l}\text { Referencia } \\
0,8(0,4-1,7)\end{array}$ & 0,6 & & \\
\hline $\begin{array}{l}\text { Tratamiento ATM empírico apropiad } \\
\text { Al menos } 1 \text { ATM activo } \\
\text { Ningún ATB plenamente activo }\end{array}$ & $\begin{array}{c}8 / 40(20 \%) \\
24 / 88(27,3 \%)\end{array}$ & $\begin{array}{l}\text { Referencia } \\
1,5(0,6-3,7)\end{array}$ & 0,4 & $\begin{array}{l}\text { Referencia } \\
1,5(0,6-4)\end{array}$ & 0,4 \\
\hline $\begin{array}{l}\text { Tratamiento ATM definitivo apropiac } \\
\text { Al menos } 1 \text { ATM activo } \\
\text { Ningún ATM plenamente activo }\end{array}$ & $\begin{array}{c}25 / 123(20,3 \%) \\
6 / 21(28,6 \%)\end{array}$ & $\begin{array}{l}\text { Referencia } \\
1,6(0,6-4,5)\end{array}$ & 0,4 & $\begin{array}{l}\text { Referencia } \\
1,9(0,6-6,5)\end{array}$ & 0,3 \\
\hline $\begin{array}{l}\text { Bacteriemia } \\
\text { Sí } \\
\text { No }\end{array}$ & $\begin{array}{c}9 / 41(22,0 \%) \\
29 / 120(24,2 \%)\end{array}$ & $\begin{array}{l}0,9(0,4-2,1) \\
\text { Referencia }\end{array}$ & 0,8 & & \\
\hline $\begin{array}{l}\text { Shock séptico } \\
\text { Sí } \\
\text { No }\end{array}$ & $\begin{array}{c}16 / 36(44,4 \%) \\
22 / 127(17,03 \%)\end{array}$ & $\begin{array}{l}3,8(1,7-8,5) \\
\text { Referencia }\end{array}$ & 0,001 & $\begin{array}{l}4,2(1,5-11) \\
\text { Referencia }\end{array}$ & 0,005 \\
\hline
\end{tabular}

EPC: enterobacterias productoras de carbapenemasas. RIC: rango intercuartil. OR: Odd Ratio. IC: intervalo de confianza. 
primer mes, incluyó sólo la presencia de shock séptico al diagnóstico (OR 4,2; IC 95\% 1,5-11). No se pudo identificar asociación entre la idoneidad del tratamiento antimicrobiano (empírico o definitivo) y la mortalidad. El área bajo la curva de la curva ROC del modelo final fue 0,69 (IC 95\% 0,58-0,8) (Figura 1).

En el supuesto de haber considerado como antibioterapia apropiada la inclusión de dos o más fármacos con actividad in vitro, el resultado del análisis multivariante resultaba similar, identificando únicamente asociación entre la mortalidad a 30 días y el shock séptico (datos no mostrados).

\section{Discusión}

Nuestro trabajo caracteriza los datos clínicos obtenidos de una amplia cohorte de pacientes con infecciones producidas por EPC en un hospital de tercer nivel en España, así como las variables relacionadas con la mortalidad. Se ha observado un importante aumento en los aislamientos de EPC en nuestro centro a partir de 2015. Del total de aislados de $K$. pneumoniae, 5,5 y 5,6\% fueron productoras de carbapenemasas en 2013 y 2014, respectivamente, frente a $24 \%$ en 2015 . Llama la atención en nuestro estudio la predominancia de OXA-48 sobre otros tipos de carbapenemasas. Este tipo de carbapenemasa es la más frecuente en España (con diferencias en distintas áreas) y en Europa, a diferencia de lo que ocurre en otros muchos países, como E.U.A., en que predomina el tipo KPC 9 .

Respecto a los factores de riesgo asociados al desarrollo de infecciones por EPC, nuestros resultados apuntan en la misma línea que trabajos previos, señalando la influencia de la edad, el grado de gravedad del proceso actual, el ingreso en unidades de cuidados críticos, la exposición previa a antimicrobianos (especialmente carbapenémicos, quinolonas y cefalosporinas) y procedimientos invasivos ${ }^{11}$.

En nuestra cohorte, la mortalidad a los 30 días afectó prácticamente a uno de cada cuatro pacientes que presentaron un proceso infeccioso por EPC. Estos hallazgos son similares a los del trabajo realizado por de Maio Carrilho y cols. ${ }^{21}$, en una cohorte prospectiva de 127 pacientes en E.U.A., en la que la mortalidad a los 30 días se situó en $28,3 \%$. En cuanto a la posibilidad de peores resultados pronósticos en las infecciones por estos microorganismos, concluyó que los pacientes infectados por $K$. pneumoniae productora de carbapenemasa presentaban una mortalidad significativamente superior comparada con las cepas sensibles (OR cruda 2,80).

Un aspecto relevante en este escenario es la relación entre la resistencia a antimicrobianos y la mortalidad, debido a la tendencia a que estos pacientes reciban un esquema terapéutico empírico inapropiado. La literatura médica previa aporta resultados contradictorios. Algunos

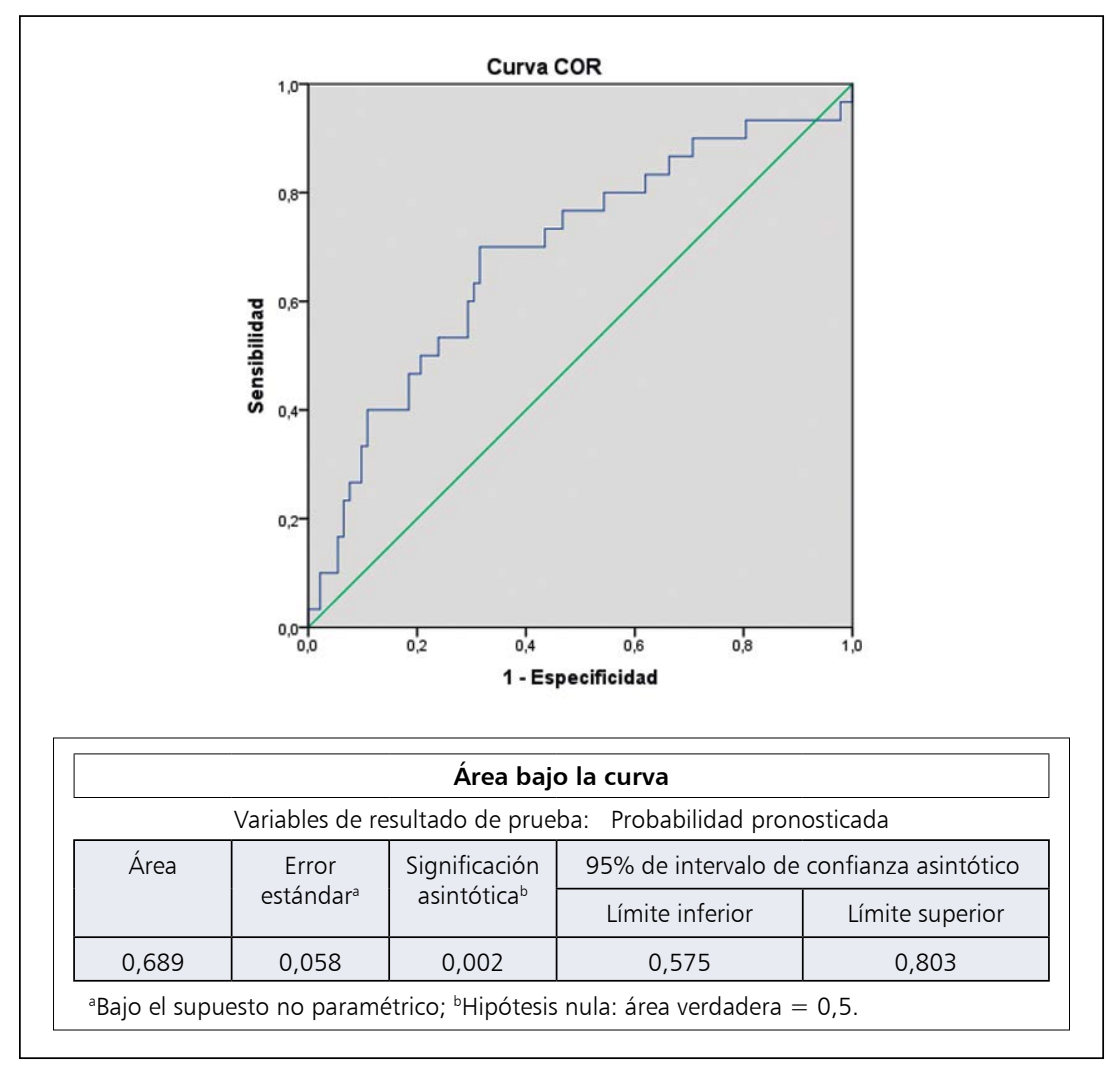

Figura 1. Curva ROC de mortalidad a 30 días.

trabajos no encontraron peores resultados cuando los agentes implicados eran microorganismos productores de $\mathrm{BLEE}^{22,23}$, ni en el caso de cepas productoras de carbapenemasas ${ }^{24}$. Sin embargo, otras publicaciones reportaron que la posibilidad de recibir antibioterapia empírica incorrecta es cuatro veces mayor en las infecciones producidas por EPC respecto a las cepas sensibles, así como una asociación significativa entre antibioterapia empírica inadecuada y mortalidad ${ }^{25}$.

En nuestro estudio, $69 \%$ de los pacientes no recibieron antimicrobiano activo alguno en la pauta empírica y $15 \%$ ninguno en el régimen definitivo; sin embargo, no encontramos asociación entre el tratamiento inapropiado y la mortalidad a los 30 días. El desarrollo de trabajos prospectivos con un adecuado diseño para mitigar la influencia de los factores de confusión aportará luz en los próximos años, como, por ejemplo, el reclutamiento de la cohorte europea que investiga sobre los factores de riesgo, el manejo clínico y sus resultados en pacientes hospitalizados por infecciones urinarias complicadas, intra-abdominales, respiratorias y bacteriemias causadas por $\mathrm{EPC}^{26}$.

Por otra parte, destacamos que aproximadamente la mitad de nuestros pacientes recibió un esquema basado 
en monoterapia. Estas cifras están en relación con que la infección urinaria fue el tipo de infección predominante, recibiendo monoterapia dos tercios de los sujetos. Sin embargo, más de $60 \%$ de los casos más graves, con bacteriemia, shock séptico o ingreso en UCI, fueron tratados con antibioterapia combinada. En trabajos previos como el de Tumbarello y cols. ${ }^{29}$, también se observó una tendencia a que los regímenes con monoterapia se aplicasen más frecuentemente en pacientes con infecciones urinarias (considerándose éstas en general, de menor gravedad), mientras que la terapia combinada era más común en pacientes de unidades de críticos. Hasta el momento, no disponemos de ensayos clínicos aleatorizados que comparen monoterapia frente a terapia combinada en las infecciones producidas por EPC, persistiendo la duda de si la monoterapia con un agente activo podría ser una opción apropiada en el caso de las infecciones urinarias ${ }^{11}$. Sin embargo, en la cohorte europea INCREMENT de bacteriemias por EPC, la antibioterapia combinada mejoró la supervivencia en los pacientes de mayor riesgo (según la escala propuesta), sin demostrar diferencias con la monoterapia en los casos de bajo riesgo de mortalidad ${ }^{30}$.

Hay que reseñar que en el período de nuestro estudio ningún paciente había recibido ceftazidima/avibactam, nuevo antimicrobiano con actividad frente a EPC tipo OXA-48 y con muy buenos resultados en estudios recientes ${ }^{23}$.

Nuestro trabajo presenta algunas limitaciones. En primer lugar, la dificultad en la recogida de datos por tratarse de un estudio retrospectivo (por ejemplo, no se recogió el tiempo desde el aislamiento hasta la primera dosis de antibiótico, empírico o definitivo), por falta de consistencia en las historias clínicas), así como la ausencia de un grupo control que permitiese reducir los posibles sesgos. No fue posible recoger los valores de cada variable en todos los pacientes, no se recogió el tiempo desde el aislamiento hasta la primera dosis de antimicrobiano (empírico o definitivo), ni se emplearon escalas de gravedad, por falta de consistencia en las historias clínicas. En segundo lugar, el limitado número de criterios de inclusión conduce a una cohorte heterogénea. Por último, debe considerarse también el posible impacto de la co-expresión de BLEE en la epidemiología y los hallazgos clínicos.

\section{Conclusiones}

Encontramos una frecuencia inesperadamente alta de tratamiento antimicrobiano inapropiado y un alto porcentaje de pacientes tratados en monoterapia. Aunque en nuestro trabajo no encontramos asociación entre el tratamiento antimicrobiano inapropiado y la mortalidad, ante la sospecha de infección por EPC es recomendable el tratamiento empírico combinado, especialmente en las infecciones graves, para aumentar la probabilidad de que los pacientes reciban al menos un fármaco activo. Son necesarios estudios prospectivos para aclarar si es necesario el tratamiento antimicrobiano combinado en las infecciones por EPC o si en infecciones leves podría ser suficiente la monoterapia.

Agradecimientos: A Jesús Sanz y Cristina Sarriá su lectura crítica del manuscrito. Asimismo, estamos en deuda con Julia Fernández por compartir los datos clínicos de algunos de los pacientes incluidos, y con Lorena Vega por su apoyo en el análisis estadístico.

\section{Referencias bibliográficas}

1.- Nordmann P, Naas T, Poirel L. Global spread of carbapenemase producing Enterobacteriaceae. Emerg Infect Dis. 2011; 17(10): 1791-8. doi: 10.3201/eid1710.110655.

2.- Walsh T R. Emerging carbapenemases: A global perspective. Int J Antimicrob Agents 2010; 36 (3): S8-14. doi: 10.1016/S09248579(10)70004-2.

3.- European Centre for Disease Prevention and Control. Antimicrobial resistance surveillance in Europe 2018. Annual Report of the European Antimicrobial Resistance Surveillance Network (EARS-Net). Stockholm: ECDC; 2019.

4.- Tórtola M T, Lavilla S, Miró E, González J J, Larrosa N, Sabaté M, et al. First detection of a carbapenem-hydrolyzing metalloenzyme in two Enterobacteriaceae isolates in Spain.
Antimicrob Agents Chemother. 2005; 49 (8): 3492-4. doi: 10.1128/AAC.49.8.34923494.2005

5.- Tato M, Coque T M, Rucz-Garbajosa $P$, Pintado V, Cobo J, Sader H S, et al. Complex clonal and plasmid epidemiology in the first outbreak of Enterobacteriaceae infection involving VIM-1 metallo-lactamase in Spain: Toward endemicity? Clin Infect Dis 2007; 45 (9): 1171-8. doi: 10.1086/522288.

6.- Tzouvelekis L S, Markogiannakis A, Psichogiou M, Tassios P T, Daikos G L. Carbapenemases in Klebsiella pneumoniae and other Enterobacteriaceae: An evolving crisis of global dimensions. Clin Microbiol Rev. 2012; 25 (4): 682-707. doi: 10.1128/ CMR.05035-11.

7.- Palacios-Baena Z R, Oteo J, Conejo C, Larrosa M N, Bou G, Fernández-Martínez M, et al. Comprehensive clinical an epidemiological assessment of colonisation an infection due to carbapenemase-producing Enterobacteriaceae in Spain. J Infect. 2016; 72 (2): 152-60. doi: 10.1016/j.jinf.2015.10.008

8.- Sánchez-Romero I, Asensio A, Oteo J, Muñoz-Algarra M, Isidoro B, Vindel A, et al. Nosocomial outbreak of VIM-1-producing Klebsiella pneumoniae Isolates of multilocus sequence type 15: molecular basis, clinical risk factors, and outcome. Antimicrob Agents Chemother 2012; 56 (1): 420-7. doi: 10.1128/ AAC.05036-11.

9.- Oteo J, Ortega A, Bartolomé R, Bou G, Conejo C, Fernández-Martínez M, et al. Prospective multicenter study of carbapenemase-producing Enterobacteriaceae from 83 hospitals in Spain reveals high in vitro susceptbility to colistin and meropenem. Antimicrob Agents Chemother 2015; 59 (6): 3406-12. doi: 10.1128/AAC.00086-15. 
S A, Trivette S L, Briggs J P, et al. Health care-associated bloodstream infections in adults: a reason to change the accepted definition of community-acquired infections. Ann Intern Med 2002; 137 (10): 791-7. doi: 10.7326/00034819-137-10-200211190-00007.

17.- EUCAST guidelines for detection of resistance mechanisms and specific resistances of clinical and/or epidemiological importance. Version 1.0, December 2013. Disponible en: http:// www.eucast.org/fileadmin/src/media/PDFs/ EUCAST_files/Resistance_mechanisms/ EUCAST detection of resistance mechanisms_v1.0_20131211.pdf (noviembre de 2017, último acceso).

18.- Asociación Médica Mundial. Declaración de Helsinki de la AMM. Principios éticos para la investigación médica en seres humanos. [citado 26 en octubre de 2017]; Disponible en: http://www.isciii.es/ISCIII/es/contenidos/ $\mathrm{fd}$-investigacion/fd-evaluacion/fd-evaluacionetica-investigacion/Declaracion-Helsinki2013-Esp.pdf.

19.- Real Decreto 1720/2007, de 21 de diciembre, por el que se aprueba el Reglamento de desarrollo de la Ley Orgánica 15/1999, de 13 de diciembre, de protección de datos Boletín Oficial del Estado, núm. 17, de 19 de enero de 2008, pp. 97858 a 97921. Disponible en: https:/www.boe.es/buscar/pdf/2008/BOE-A2008-979-consolidado.pdf.

20.- De Maio Carrilho C M D, de Oliveira L M, Gaudereto J, Perozin J S, Urbano M R, et al. A prospective study of treatment of carbapenemresistant Enterobacteriaceae infections and risk factors associated with outcome. BMC Infect Dis 2016; 16: 629. http://doi. org/10.1186/s12879-016-1979-z.

21.- Xu L, Sun X, Ma X. Systematic review and meta-analysis of mortality of patients infected with carbapenem-resistant Klebsiella pneumoniae. Ann Clin Microbiol Antimicrob 2017; 16 (1): 18. doi: 10.1186/s12941-0170191-3.
22.- Bhavnani S M, Ambrose P G, Craig W A, Dudley M N, Jones R N, SENTRY Antimicrobial Surveillance Program. Outcomes evaluation of patients with ESBLand non-ESBL-producing Escherichia coli and Klebsiella species as defined by CLSI reference methods: report from the SENTRY Antmicrobial Surveillance Program. Diagn Microbiol Infect Dis 2006; 54 (3): 231-6. doi: 10.1016/j.diagmicrobio.2005.09.011.

23.- Kim B N, Woo J H, Kim M N, Ryu J, Kim Y S. Clinical implications of extendedspectrum beta-lactamase-producing Klebsiella pneumoniae bacteraemia. J Hosp Infect 2002; 52 (2): 99-106. doi: 10.1053/jhin.2002.1288.

24.- García-Sureda L, Doménech-Sánchez A, Barbier M, Juan C, Gascó J, Albertí S. OmpK26, a novel porin associated with carbapenem resistance in Klebsiella pneumoniae. Antimicrob Agents Chemother 2011; 55 (10): 4742-7. doi: 10.1128/ AAC.00309-11

25.- Zilberberg M D, Nathanson B H, Sulham K, Fan W, Shorr A F. Carbapenem resistance, inappropriate empiric treatment and outcomes among patients hospitalized with Enterobacteriaceae urinary tract infection, pneumonia and sepsis. BMC Infect Dis. 2017; 17: 279. doi: 10.1186/s12879-017-2383-z.

26.- Gutiérrez-Gutiérrez B, Sojo-Dorado J, BravoFerrer J, Cuperus N, Kraker M De, Kostyanev $\mathrm{T}$, et al. EUropean prospective cohort study on Enterobacteriaceae showing REsistance to CArbapenems (EURECA): a protocol of a European multicentre observational study. BMJ Open, 20177 (4): e015365. doi: 10.1136/ bmjopen-2016-015365.

27.- Tumbarello M, Viale P, Viscoli C, Trecarichi E M, Tumietto F, Marchese A, et al. Predictors of mortality in bloodstream infections caused by Klebsiella pneumoniae carbapenemaseproducing $K$. pneumoniae: importance of combination therapy. Clin Infect Dis 2012; 55 (7): $943-50$. doi: $10.1093 / \mathrm{cid} / \mathrm{cis} 588$. 